UVX 2010 (2011) 77-81

DOI: $10.1051 / \mathrm{uvx} / 2011011$

(C) Owned by the authors, published by EDP Sciences, 2011

\title{
Optiques spatiales pour les surveys en astronomie UV
}

\author{
R. Grange
}

\author{
Laboratoire d'Astrophysique de Marseille, 38 rue Fréderic Joliot-Curie, 13388 Marseille, \\ France
}

\begin{abstract}
Résumé. Les optiques spatiales dédiées à l'astronomie UV doivent être optimisées pour collecter un flux UV extrêmement faible comparé aux sources mises en œuvre dans nos laboratoires. Cet objectif se heurte à une série de difficultés spécifiques au domaine UV : réflectivité et transmissivité modestes des matériaux, reflets parasites élevés, forte dépendance des indices avec la longueur d'onde, difficulté de réalisation des revêtements de surface efficaces. Cette situation conduit à privilégier les systèmes catadioptriques avec un nombre de surfaces optiques minimal tout en conservant une bonne correction instrumentale. L'instrument d'optique type en astronomie UV est ainsi caractérisé par peu d'éléments optiques avec pour chacun une grande complexité de réalisation (forme, revêtement de surface, microstructure), et souvent un champ important et une ouverture numérique élevée. La description de trois expériences UV menées au LAM, FUSE puis GALEX en satellite et FIREBALL en ballon stratosphérique, illustrera ce principe de sobriété du concept optique.
\end{abstract}

\section{INTRODUCTION}

Le laboratoire d'Astrophysique de Marseille (LAM) a été dès sa fondation en 1967 impliqué dans des expériences spatiales (ballons, fusées, satellites) seules capables de donner accès aux radiations UV bloquées par l'atmosphère. L'astronomie UV (90 à $350 \mathrm{~nm}$ ) permet en effet de mesurer la température et la composition des étoiles jeunes et chaudes et fournit ainsi une information précieuse sur le taux de formation stellaire et sur l'évolution des galaxies.

En instrumentation spatiale le design optique est fortement contraint par le volume limité du véhicule et une masse requise faible. De plus l'environnement vibratoire et thermique sévère ainsi qu' une grande exigence de fiabilité conduisent à une simplification de l'architecture instrumentale en minimisant le nombre de canaux et de mécanismes.

Le domaine UV spatial y ajoute un certain nombre de contraintes spécifiques. L'UV se caractérise par un nombre réduit de matériaux transparents $(\mathrm{MgF} 2, \mathrm{CaF} 2)$, allié à une forte variation de leurs indices optiques qui rend difficile les corrections des aberrations chromatiques. Les optiques en réflexion sont donc privilégiées tout en maintenant un nombre d'éléments optiques réduit pour une efficacité maximum. On aboutit souvent à des optiques aux formes complexes à réaliser et intégrant plusieurs fonctions.

En plus des études photométriques utilisant en général des imageurs, les études spectrales qui donnent accès aux paramètres physiques des sources astronomiques sont essentielles en astronomie UV ; elle est en général réalisée au moyen de réseaux de diffraction. Ces derniers sont préférés par rapport aux prismes en raison de leur plus grand pouvoir de dispersion et du fait que peu de matériaux sont transparents en UV.

This is an Open Access article distributed under the terms of the Creative Commons Attribution-Noncommercial License 3.0, which permits unrestricted use, distribution, and reproduction in any noncommercial medium, provided the original work is properly cited. 


\section{LE SPECTROGRAPHE DE FUSE}

L'expérience FUSE (Far Ultraviolet Spectroscopic Explorer) est un spectrographe en UV lointain (90$120 \mathrm{~nm})$ avec une haute résolution spectrale $(\mathrm{R}=30000)$ lancé par la NASA en juin 1999. Pour atteindre cette haute résolution spectrale avec un minimum d'optique, le schéma optique (fig. 1) est basé sur un réseau de diffraction à haute densité de traits $(5767 \mathrm{t} / \mathrm{mm})$ en montage de Rowland. Cependant à l'ouverture numérique de travail $(\mathrm{F} / 4.5)$ choisie pour maximiser la surface collectrice, les aberrations de coma et d'astigmatisme deviennent prépondérantes et leur correction est nécessaire pour atteindre la résolution spectrale requise. Pour ne pas ajouter une surface correctrice supplémentaire, nous avons fait appel à la correction des aberrations par holographie [1-3].

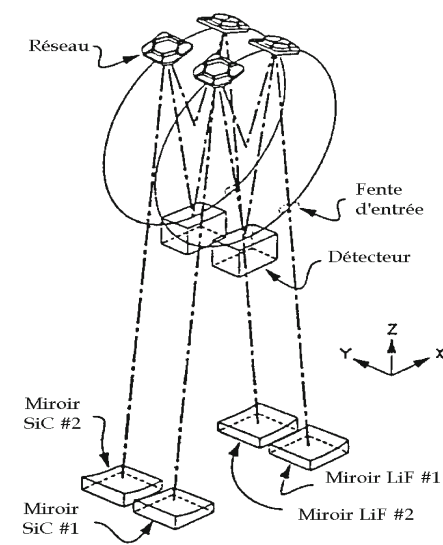

Figure 1. Schéma optique du spectrographe FUSE.

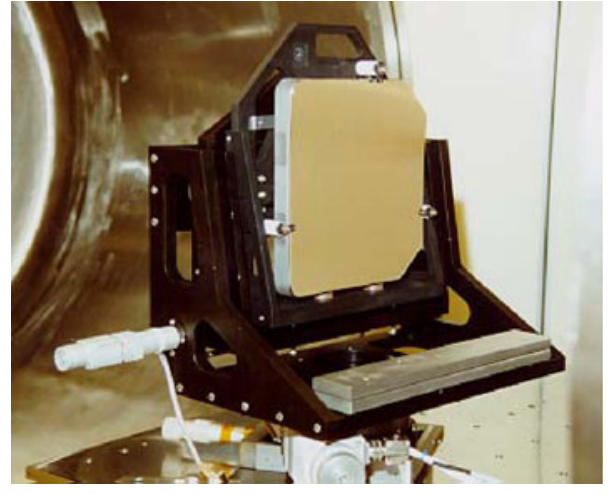

Figure 2. Réseau FUSE en cours de calibration au LAM.

L'enregistrement holographique d'un réseau de diffraction consiste à imprimer à la surface du composant optique le champ de franges d'interférences produit par deux ondes cohérentes. En introduisant des aberrations dans le système d'enregistrement, le réseau ainsi produit a la capacité de corriger lors de son utilisation les aberrations inhérentes au système sans ajouter de surfaces supplémentaires. L'originalité du réseau de FUSE (fig. 2) réside dans une nouvelle géométrie d'enregistrement holographique produisant des ondes aberrées grâce à deux sources stigmatiques dont l'une est virtuelle. Il est à noter que ce type d'enregistrement a été également utilisé pour le Cosmic Origins Spectrograph installé récemment par la navette spatiale sur le Hubble Space Telescope.

\section{LE GRISM DE GALEX}

La mission GALEX (Galaxy Evolution Explorer) est un spectro-imageur en UV dédié à retracer l'évolution des galaxies grâce à un survey de tout le ciel en imagerie et en spectroscopie [4,5].

Pour combiner sans mécanisme très complexe les deux canaux d'imagerie et de spectroscopie nous avons proposé l'utilisation d'un grism qui a la propriété de ne pas dévier le faisceau incident et de disperser la lumière autour d'une longueur d'onde centrale dite de blaze. Un grism est réalisé en répliquant un réseau de diffraction maitre sur l'hypoténuse d'un prisme. Ce procédé classique de réplique utilisant une résine ne peut pas être appliqué en UV en raison de la forte absorption du film de résine résiduel. Nous avons développé avec la société Jobin-Yvon une méthode de gravure directe dans le substrat en $\mathrm{CaF} 2$ grâce au transfert par usinage ionique du profil préalablement gravé dans une 
couche métallique. Les efficacités effectivement mesurées dans les ordres 1 et 2 sont respectivement de $80 \%$ et $60 \%$. La fig. 3 montre le profil blazé du réseau obtenu au microscope à effet tunnel.

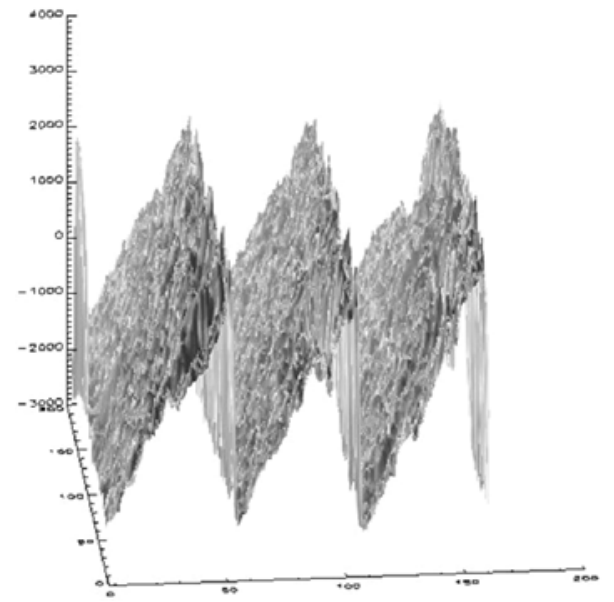

Figure 3. Profil du réseau au STM.

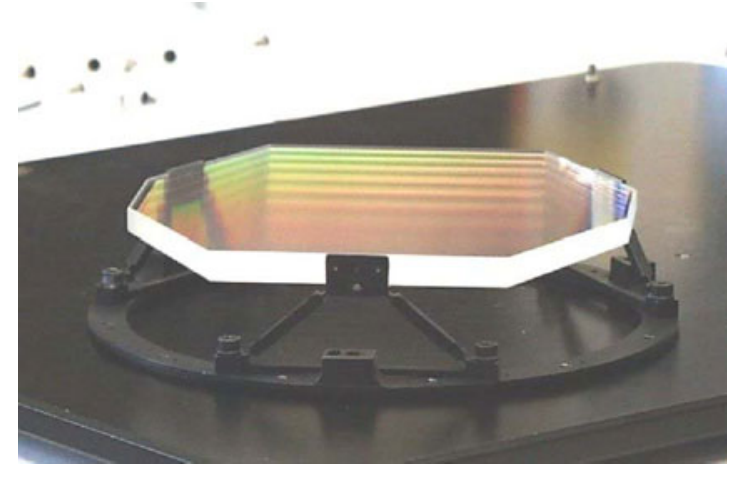

Figure 4. Grism Galex avec sa monture de vol.

Le CaF2 est un matériau très fragile et une monture quasi statique (fig. 4) avec des bipodes en Titane a été étudiée et réalisée pour résister aux niveaux de vibrations aléatoires exigés (15 G RMS).

\section{LES OPTIQUES DE FIREBALL}

Fireball est une mission ballon (fig. 5) exploratrice pour détecter pour la première fois le fond diffus du milieu intergalactique pour une bande de redshift $\mathrm{Z}$ comprise entre 0.3 et 1.0 . Les vols ballons stratosphériques à $35 \mathrm{~km}$ d'altitude [6] donnent accès à une fenêtre UV (190-215 nm) avec un très faible fond diffus et sont ainsi des approches alternatives aux missions satellites.

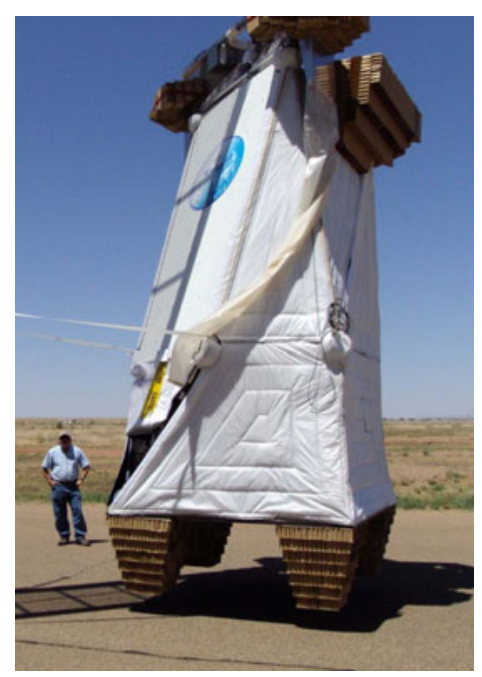

Figure 5. Nacelle Fireball en phase de préparation au lancement. 
Le besoin combiné de résolution spatiale et d'une grande sensibilité aux sources diffuses conduit à un télescope de 1 mètre de diamètre qui alimente un spectrographe intégral de champ à grande ouverture numérique $(\mathrm{F} / 2.5)$.

Au lieu de pointer directement le télescope et son spectrographe, nous avons opté pour un pointage à faible inertie dynamique basé sur un sidérostat qui réfléchit la lumière de l'objet étudié vers le télescope fixe dans la structure nacelle. Pour simplifier l'architecture, la structure nacelle fait également office de structure du télescope [7]. Comme le champ de Fireball est relativement réduit (3 minutes d'arc) l'optique du télescope peut se réduire à un simple miroir parabolique ouvert à F/2.5.

Le spectrographe intégral [8] de champ utilise un faisceau de 400 fibres optiques qui groupées à son entrée découpent régulièrement le champ de 3 minutes d'arc au foyer du parabolique. A l'autre extrémité du faisceau, les fibres sont alignées pour former une longue fente de $60 \mathrm{~mm}$ de haut qui sera la fente d'entrée de la chambre spectroscopique. Pour minimiser le nombre de surfaces, cette chambre spectroscopique est basée sur un montage Offner utilisé en Littrow (fig. 6) qui comporte seulement un miroir sphérique et un réseau convexe. Malgré la haute densité de traits $(5000 \mathrm{t} / \mathrm{mm})$ du réseau holographique (fig. 7) celui-ci ne fonctionne pas en régime purement électromagnétique et l'optimisation de son traitement de surface a nécessité une modélisation fine en s'appuyant sur l'héritage des grisms du Cosmic Origins Spectrograph. Nous avons obtenu une efficacité de $35 \%$ à $200 \mathrm{~nm}$ pour un réseau convexe de $230 \mathrm{~mm}$ de diamètre.

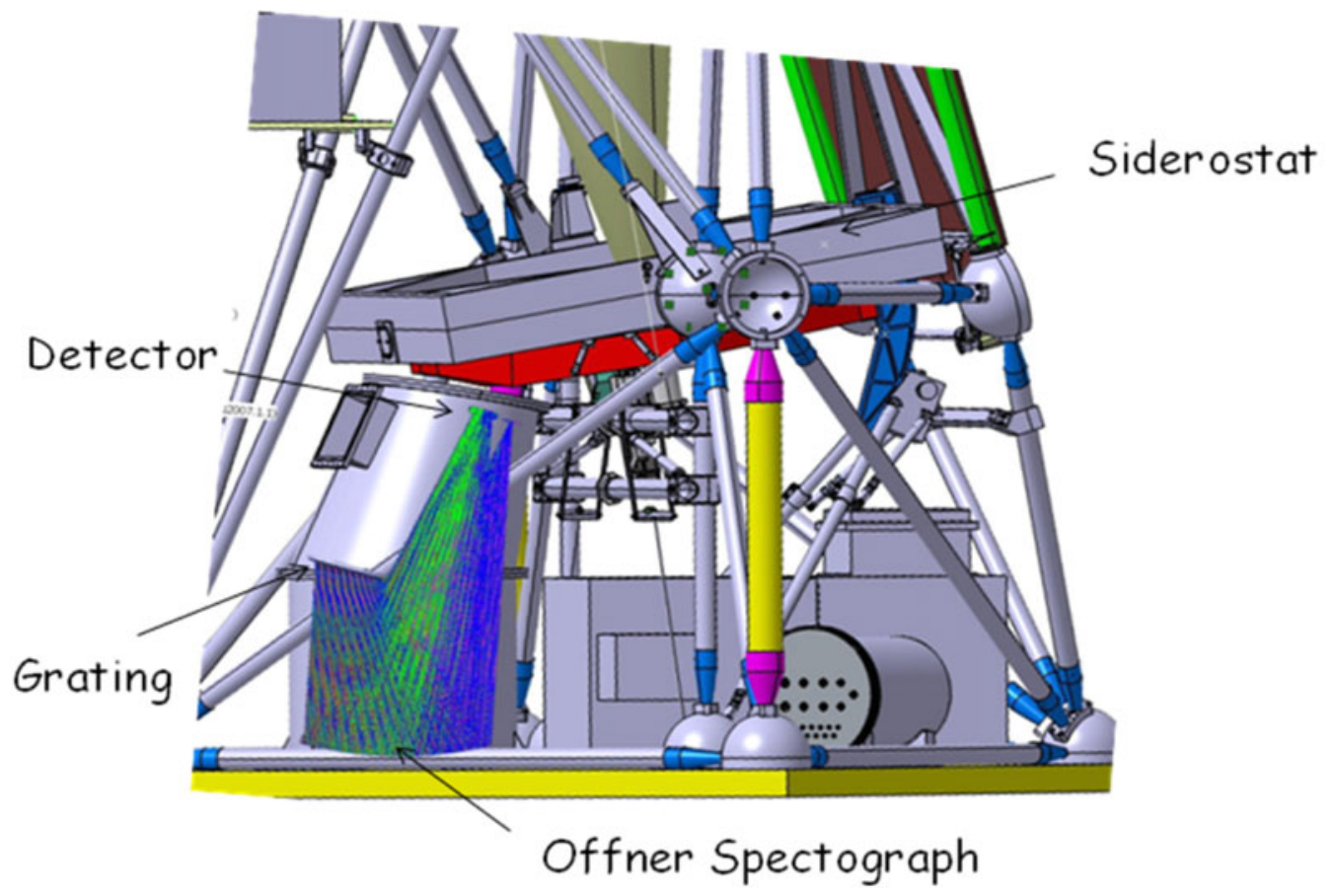

Figure 6. Vue du sidérostat et de la chambre spectroscopique basée sur un montage Offner Littrow.

\section{CONCLUSION}

La description de trois missions UV (FUSE, GALEX, FIREBALL) montre la spécificité du domaine UV spatial qui est caractérisé par une architecture optique avec peu de surfaces et peu de mécanismes, des systèmes essentiellement à miroirs et des spectrographes basés sur des réseaux de diffraction souvent 


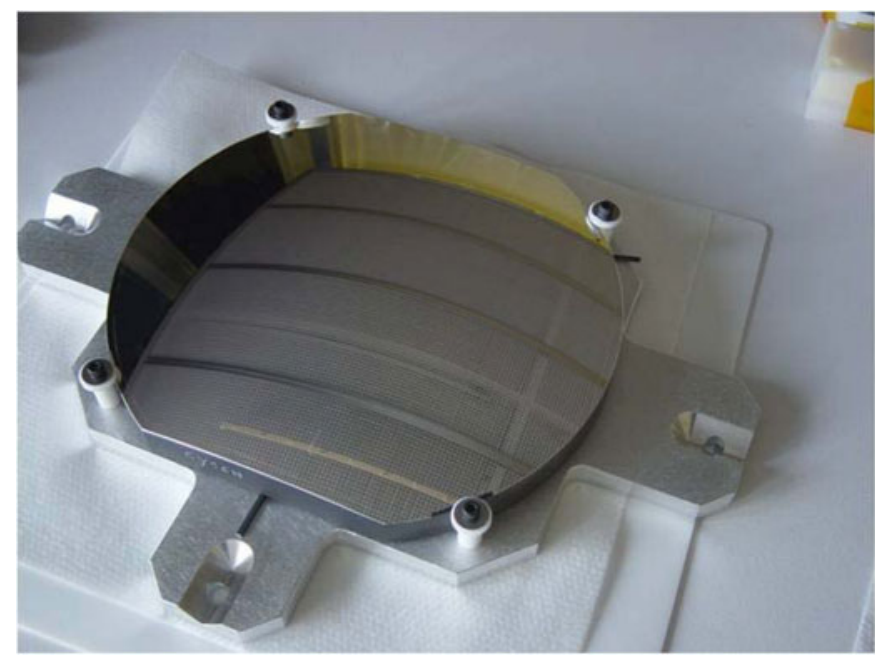

Figure 7. Réseau holographique de Fireball à 5000 t/mm (diamètre $230 \mathrm{~mm}$ ).

corrigés des aberrations. Les optiques sont donc complexes à réaliser soit par leur forme très asphérique ou par le fait d'intégrer des corrections d'aberrations. Dans le domaine des systèmes diffractifs, les nouvelles techniques de photolithographie (faisceau d'électron ou écriture laser) développées pour la microélectronique sont très prometteuses par leur facilité d'intégrer une fonction de phase quelconque pour corriger les aberrations du système optique.

\section{Références}

[1] R. Grange "Holographic spherical gratings: a new family of quasi-stigmatic designs for the Rowland-circle mounting,' 32, 4875-4880 (1993)

[2] R. Grange "Aberration reduced holographic spherical gratings for Rowland circle spectrographs," Appl. Opt. 31, 3744-3749 (1992)

[3] R. Grange, M. Laget, "Holographic diffraction gratings generated by aberrated wave fronts Application to a high-resolution far-ultraviolet spectrograph", Appl. Opt. 30, 3744-3749 (1991)

[4] Martin, D. Christopher et al "The Galaxy Evolution Explorer: A Space Ultraviolet Survey Mission", UCP, L1-L6, 2005

[5] R. Grange, B. Milliard, J. Flamand, A. Pauget, G Waultier, G. Moreaux, C. Rossin, M. Viton, M. Neviere, « Galex UV Grism for Slitless Spectoscopy Survey », ICSO 2000, 4th International Conference on Space Optics

[6] Grange, R., Evrard, J., Milliard, B., Martin, C., Schiminovich, D., Mirc, F., Matuszewski, M., Tuttle, S., Rahman, S., Deharveng, J., and 3 coauthors FIREBall Instrument Overview, AAS Meeting in Washington January 2010

[7] Rossin, C., Grange, R., Milliard, B., Martin, L., Moreaux, G., Blanchard, P., Deharveng, J.-M., Evrard, J., Martin, C., McLean, R., and Schiminovich, D., "Semi-kinematic mount of the FIREBALL large optics", SPIE, 2008

[8] Grange Robert, Milliard Bruno, McLean Ryan, Martin Christopher, Deharveng Jean Michel, and Schiminovich David "Three-dimensional spectroscopy with a fiber-fed NUV spectrograph", SPIE, 308-312, 2005 